\title{
Dialogical Consciousness and Descriptive Experience Sampling: Implications for the Study of Intrapersonal Communication in Sport
}

\author{
Judy L. Van Raalte ${ }^{1,2 *}$, Andrew Vincent ${ }^{3}$ and Yani L. Dickens ${ }^{4}$ \\ ${ }^{1}$ Department of Psychology, Springfield College, Springfield, MA, United States, ${ }^{2}$ College of Health Sciences, Wuhan Sports \\ University, Wuhan, China, ${ }^{3}$ Counseling Center, SUNY Oneonta, Oneonta, NY, United States, ${ }^{4}$ Counseling Services, \\ University of Nevada, Reno, NV, United States
}

Keywords: athlete, inner experience, open-beginninged methods, presupposition, self-talk

OPEN ACCESS

Edited by:

Thomas M. Brinthaupt, Middle Tennessee State University, United States

Reviewed by:

Hubert Hermans,

Radboud University Nijmegen,

Netherlands

James Hardy,

Bangor University, United Kingdom

${ }^{*}$ Correspondence:

Judy L. Van Raalte

jvanraa/@springfieldcollege.edu

Specialty section

This article was submitted to

Cognitive Science,

a section of the journal

Frontiers in Psychology

Received: 13 January 2019 Accepted: 08 March 2019

Published: 03 April 2019

Citation:

Van Raalte JL, Vincent $A$ and

Dickens YL (2019) Dialogical

Consciousness and Descriptive

Experience Sampling: Implications for

the Study of Intrapersonal

Communication in Sport.

Front. Psychol. 10:653.

doi: 10.3389/fpsyg.2019.00653
Inner experience and intrapersonal communication research in sport psychology has been largely dominated by a focus on self-talk, which has typically been examined using retrospective self-report measures. Although the existing self-talk literature has addressed aspects of athlete's inner experience, attempts to extend the theoretical scope of intrapersonal communication in sport has been limited by an adherence to linear, causal models of self-talk, as well as by methodological challenges associated with assessing inner experience. The purpose of this paper is to present theoretical and methodological approaches that can be used for further understanding of intrapersonal communication and inner experience in sport. The paper begins with a brief history of sport self-talk theory and research. Next, a discussion of dialogical self (Hermans et al., 1992; Hermans and Hermans-Konopka, 2010) and dialogical consciousness (Larrain and Haye, 2012; Haye and Larrain, 2013) as they relate to sport self-talk theory is presented. Descriptive Experience Sampling (DES), a promising method for exploring inner experience and self-talk in sport is described. We conclude with suggestions related to integrating dialogical theories and DES into the study of intrapersonal communication in sport.

\section{HISTORY OF SELF-TALK IN SPORT PSYCHOLOGY}

Examining the origins and history of self-talk research in sport psychology provides important insight into strengths and limitations of the literature. Early sport psychology self-talk research primarily involved linear experimental designs that assessed the effects of assigned self-talk on laboratory-based motor learning and motor performance tasks (Landers, 1995). These experimental approaches required self-talk phrases to be categorized, so that hypotheses about how types of self-talk affect learning and performance could be tested. Although linear, causal theories can provide insight related to the effects of self-talk on certain tasks, it is not possible to answer questions such as "How do athletes experience their own self-talk?" "What is the purpose of self-talk in sport?" and "How does self-talk work?" through categorization and experimental testing alone.

The self-talk literature was subsequently shaped by cognitive and cognitive behavioral theories (CBT) of Ellis (1957) and Beck (1975), which focused on self-talk as emblematic of deeply held "core beliefs" related to self-esteem, confidence, self-concept, and self-efficacy. Although cognitive behavioral paradigms advanced the application of mental skills interventions, such theoretical approaches were limited by their conceptualization of the self as autonomous, unitary, and self-contained (Hermans and Hermans-Konopka, 2010). For instance, the assumption that an athlete's critical self-statement reflects low self-esteem leaves little room for the experience of inner conflict (an athlete who oscillates between positive and negative self-concept) or self-talk 
that echoes the voice of some important other (an athlete hearing a coach saying "that's not good enough" in their head). Researchers who consider self-talk in a broader paradigmatic context and apply methods that circumvent the limitations of retrospective self-report, may inspire new inquiry and advance understanding of inner experience and self-talk.

\section{EXPANDING THEORY IN INTRAPERSONAL COMMUNICATION: DIALOGICAL CONSCIOUSNESS}

Theories from discursive psychology, especially ideas about dialogical self (Hermans et al., 1992; Hermans and HermansKonopka, 2010) and dialogical consciousness (Larrain and Haye, 2012; Haye and Larrain, 2013), provide alternative perspectives with potential for expanding current theory, research, and practice in sport psychology. Dialogical theories of self are based on philosophical assumptions of constructivism, which view the self as multifaceted, contextual, and created through interaction with the social world (Hermans et al., 1992; Hermans and Hermans-Konopka, 2010). Perhaps the most notable feature of theories of dialogical consciousness is that key aspects of inner experience are viewed as taking place in the form of a dynamic conversation that is polyphonic, consisting of many "voices" (Hermans et al., 1992; Larrain and Haye, 2012). These voices, which can be based in language, emotion, or other forms of experience, reflect different viewpoints, perspectives, or positions that might occur to a person (Puchalska-Wasyl, 2016). For example these voices might take the form of internalized Ipositions that reflect different versions of self (e.g., ideal self, undesired self, real self), internalized interlocutors who represent external figures such as a coach, a close friend, or a therapist, or norms or rules that have been internalized from culture and society (Hermans and Hermans-Konopka, 2010; PuchalskaWasyl, 2016).

Ideas pertaining to dialogical consciousness were introduced to the sport psychology self-talk literature via the sport-specific model of self-talk, which raises questions pertaining to inner discourse such as "If we already know everything we know, then why do we talk to ourselves?" and "What are we doing when we engage in self-talk?" (Van Raalte et al., 2016, pp. 140-141). Although answers to these questions cannot be understood using linear, causal models that focus on self-talk categorization, they can be addressed through the lens of dialogical self whereby intrapersonal communication is not about messages being sent and received by a singular self but rather a conversation between internalized positions taking place in the society of the mind (Hermans and Hermans-Konopka, 2010). For instance, an athlete who misses a pass may have self-talk such as "not good enough, you have to make that play" and "no worries, you can do it." If we focus solely on the content, we lose a chance to gain understanding of that athlete's internal world where the first statement may reflect the internalized voice of a critical coach or parent, and the latter may reflect the internalized voice of a mentor or a fan.
Understanding intrapersonal communication in this way opens additional avenues for research, some of which are currently under study in the area of dialogical consciousness but missing from sport psychology. For instance, Hermans (2003) has discussed the importance of power differential between Ipositions and interlocutors, suggesting that certain voices are likely to be more influential in consciousness by being more dominant in internal dialogue. In sport psychology, practitioners and researchers would benefit from better understanding which internal voices are dominant and passive and how intentionally used self-talk interacts with athletes' dominant and passive internal voices and performance.

Integrative and confrontational dialogue types present a second avenue for exploration. Integrative internal dialogues move toward synthesis and solution between internalized voices as existing positions come together as part of the construction of a new position, whereas confrontational internal dialogues accentuate difference and result in cognitive dissonance (Hermans and Hermans-Konopka, 2010; Puchalska-Wasyl, 2016, 2017). Intrapersonal communication that takes place between an athlete's inner critic and inner fan could serve as an example of this. In a confrontational dialogue, one position becomes dominant while the other is silenced; this might result in self-talk such as "ignore that positive talk, you are playing like garbage." Oppositely, an integrative dialogue would move toward a position that includes both "inner critic" and "inner fan" and may result in self-talk such as "you can finish this game strong, but let's work on that in practice next week." Exploring the extent to which integrative and confrontational dialogues occur for athletes and the ways these different types of dialogues shape athlete experiences could prove useful in understanding intrapersonal communication in sport, especially given the nature of existing applied interventions such as thought stopping and thought replacement, which employ confrontational approaches designed to silence unwanted voices in internal dialogue (Hardy and Oliver, 2014).

The connection between self, culture, and social context is a key feature of dialogical self theory, as internal dialogue is seen as a reflection of both individual experience and larger cultural forces (Hermans, 2003; Hermans and Hermans-Konopka, 2010). Viewing internal dialogue as being inextricably interconnected with the social context has important implications for selftalk in sport and could provide several avenues for future study. For instance, a given internalized position may be an internalization of a prominent cultural narrative or, in the case of sport, some aspect of team culture. This connection between social context, culture, and the internal world of an athlete stands in contrast to traditional causal, linear, category-focused, information-processing views of sport self-talk and provides a theoretical lens through which cultural differences in self-talk can be understood. Integrating theories of dialogical consciousness into existing theories of intrapersonal communication in sport can also direct applied and research attention to racism, sexism, and other oppressive forces that may be manifested as voices that play out in the internal dialogue of athlete consciousness. One of the major challenges associated with these dialogical concepts pertains to their assessment. Standardized self-report 
questionnaires are limited in capturing athletes' experiences related to dialogical processes.

\section{EXPLORING INNER EXPERIENCE: THE DESCRIPTIVE EXPERIENCE SAMPLING (DES) METHOD}

Self-talk research in sport has been constrained by the ways self-talk is studied (Hardy and Jones, 1994; Brinthaupt et al., 2015). Self-report questionnaires have traditionally served as primary sources of self-talk data, despite concerns about their validity (Van Raalte et al., 2014; Van Raalte and Vincent, 2017; Thibodeaux and Winsler, 2018), extensive evidence that these and other retrospective observations are unreliable (e.g., Brewer et al., 1991; Wells and Loftus, 2003), and the fact that recalling inner events is problematic (Hurlburt and Melancon, 1987; Koriat and Bjork, 2005). Approaches that improve upon existing methods have occasionally been used in sport and exercise psychology research, such as think-aloud methods (Fuhrer, 1985; McPherson, 1999; Whitehead et al., 2015), Ecological Momentary Assessment (EMA; Biddle et al., 2009), and the Experience Sampling Method (ESM; Cerin et al., 2001). Although each of these methods sample inner experience during sport performance, each has limitations (Dickens et al., 2018). One method that overcomes many of these shortcomings and is well-suited to the exploration of the dialogical self, dialogical consciousness, and the discursive nature of athlete's inner experiences is DES.

DES is a method that uses a random beeper to directly sample "pristine" inner experience contemporaneously and directly, circumventing many of the limitations of self-report measures and retrospection. DES is "open-beginninged," openended, and uses focused non-leading questions like "what was your inner experience, if any, at the moment of the beep" to direct participants to real-time, momentary experience. Whereas, standardized questionnaires, EMA, and ESM are often influenced by the theory of inner experience that they are designed to measure, DES brackets presuppositions to prevent experimenter expectancies from contaminating observed inner experience. DES also offers several methodological improvements that yield high fidelity samples of inner experience. For example, DES includes collections of random representative samples; intensive training to help participants observe and report inner experience; and extensive collaboration with participants around investigating their inner experience through video-recorded interviews within $24 \mathrm{~h}$ of sample collection. DES studies have shown high inter-observer reliability (Hurlburt and Heavey, 2002), DES has been validated with Functional Magnetic Resonance Imaging (fMRI) (Kühn et al., 2014), and DES has been shown to be feasible during sport performance (Dickens et al., 2018). The major cost of implementing DES is the quantityfor-quality tradeoff. DES is labor-intensive, requiring $5-10 \mathrm{~h}$ of interview time per participant (Hurlburt and Akhter, 2006; McKelvie, 2019).

DES researchers suggest that DES advances understanding of actual momentary inner experience, often yielding unique contributions. For instance, although many have presumed that self-talk is pervasive, if not ubiquitous, in activities such as silent reading or sport performance, DES research has shown that inner experience typically consists of five frequent phenomena (5FP) (Kühn et al., 2014) including inner speaking, inner seeing, sensory awareness, feeling, and unsymbolized thinking. Inner speaking is self-talk spoken silently to oneself, inner seeing is visual imagery, and sensory awareness includes bodily sensation (e.g., pain, tension, hunger), and feeling is emotion (e.g., anxiety, anger, joy). Unsymbolized thinking is a seldom recognized but explicit thought process that takes place without the presence of words or images (see Hurlburt and Akhter, 2008) and occurs about as frequently as the more well-known 5FP (Lapping-Carr and Heavey, 2017). DES research suggests that inner experience is idiosyncratic since inner experiences outside of the 5FP can and do occur, including being in a flow state and completely absorbed in an activity (Lapping-Carr and Heavey, 2017) and having no inner experience occurring at the moment of the beep (Hurlburt and Schwitzgebel, 2011). In a sport context, Dickens et al. (2018) found that inner experience during golf performance included all 5FP, speaking aloud and inner speaking both occurred during golf, self-talk was a frequent but not the predominant inner experience, inner-speaking self-talk was 6 times as frequent as speaking aloud self-talk, and effortful, intentional use of self-talk (i.e., System 2 self-talk) was rare. Also, some participants experienced no self-talk, and one participant reported no inner experience in over half of their samples, illustrating the idiosyncratic nature of inner experience during sport performance.

\section{FUTURE DIRECTIONS}

Taken together, theories of dialogical self, dialogical consciousness, and DES challenge assumptions and inspire new theorizing and research in the area of intrapersonal communication and inner experience in sport. Considering athlete experience as dialogical allows us to move beyond CBT cause-effect paradigms that focus on categorization of self-talk and explore possible theories related to I-positions and interlocutors, power dynamics, and confrontational vs. integrative inner-dialogue types. DES provides the tools necessary for precise empirical assessment of these theoretical ideas and can provide insights related to self-talk. Indeed, DES research has already shown that self-talk is a less prevalent aspect of inner experience than previously suggested in the sport psychology literature (Dickens et al., 2018). Together, theories of dialogical self, dialogical consciousness, and DES have the potential to advance theoretical and practical knowledge by validating previous findings and/or uncovering new findings.

\section{AUTHOR CONTRIBUTIONS}

All three authors developed and contributed to this work. AV developed ideas related to dialogical self and dialogical consciousness. YD developed ideas related to Descriptive Experience Sampling (DES). 


\section{ACKNOWLEDGMENTS}

The authors world like to acknowledge the theoretical, conceptual, and emotional support provided by Jessica Younger Dickens, Russell T. Hurlburt, and Rebecca Vincent. We would

\section{REFERENCES}

Beck, A. T. (1975). Cognitive Therapy and the Emotional Disorders. Madison, CT: International Universities Press.

Biddle, S. J., Gorely, T., Marshall, S. J., and Cameron, N. (2009). The prevalence of sedentary behavior and physical activity in leisure time: a study of scottish adolescents using ecological momentary assessment. Prevent. Med. 48, 151-155. doi: 10.1016/j.ypmed.2008.10.025

Brewer, B. W., Van Raalte, J. L., Linder, D. E., and Van Raalte, N. S. (1991). Peak performance and the perils of retrospective introspection. J. Sport Exerc. Psychol. 13, 227-238. doi: 10.1123/jsep.13.3.227

Brinthaupt, T. M., Benson, S. A., Kang, M., and Moore, Z. D. (2015). Assessing the accuracy of self-reported self-talk. Front. Psychol. 6:570. doi: 10.3389/fpsyg.2015.00570

Cerin, E., Szabo, A., and Williams, C. (2001). Is the experience sampling method (ESM) appropriate for studying pre-competitive emotions? Psychol Sport Exerc. 2, 27-45. doi: 10.1016/S1469-0292(00)00009-1

Dickens, Y. L., Van Raalte, J., and Hurlburt, R. T. (2018). On investigating selftalk: a descriptive experience sampling study of inner experience during golf performance. Sport Psychol. 32, 66-73. doi: 10.1123/tsp.2016-0073

Ellis, A. (1957). Rational psychotherapy and individual psychology. J. Individ. Psychol. 13, 38-44.

Fuhrer, U. (1985). Performance analysis using behavior-setting programs. Zeitschrift für Experimentelle Angewandte Psychol. 32, 194-216.

Hardy, J., and Oliver, E. J. (2014). “Thought stopping," in Encyclopedia of Sport and Exercise Psychology, eds R. C. Eklund and G. Tenenbaum (Thousand Oaks: Sage), 750-751.

Hardy, L., and Jones, G. (1994). Current issues and future directions for performance-related research in sport psychology. J. Sports Sci. 12, 61-92. doi: 10.1080/02640419408732158

Haye, A., and Larrain, A. (2013). Discursively constituted experience, or experience as reply: a rejoinder. Theory Psychol. 23, 131-139. doi: $10.1177 / 0959354312465484$

Hermans, H., and Hermans-Konopka, A. (2010). Dialogical Self Theory: Positioning and Counter-Positioning in a Globalizing Society. Cambridge: Cambridge University Press.

Hermans, H. J. M. (2003). The construction and reconstruction of a dialogical self. J. Constr. Psychol. 16, 89-130. doi: 10.1080/10720530390117902

Hermans, H. J. M., Kempen, H. J. G., and Van Loon, R. J. P. (1992). The dialogical self: beyond individualism and relationism. Am Psychol. 47, 23-33. doi: 10.1037/0003-066X.47.1.23

Hurlburt, R., and Schwitzgebel, E. (2011). Little or no experience outside of attention? J. Consciou. Studies 18:234.

Hurlburt, R. T., and Akhter, S. A. (2006). The descriptive experience sampling method. Phenomenol. Cogn. Sci. 5, 271-301. doi: 10.1007/s11097-006-9024-0

Hurlburt, R. T., and Akhter, S. A. (2008). Unsymbolized thinking. Conscious. Cogn. 17, 1364-1374. doi: 10.1016/j.concog.2008.03.021

Hurlburt, R. T., and Heavey, C. L. (2002). Interobserver reliability of descriptive experience sampling. Cognit. Ther. Res. 26, 135-142. doi: 10.1023/A:1013802006827

Hurlburt, R. T., and Melancon, S. M. (1987). How are questionnaire data similar to, and different from, thought sampling data? Five studies manipulating retrospectiveness, single-moment focus, and indeterminacy. Cognit. Ther. Res. 11, 681-704. doi: 10.1007/BF01176005

Koriat, A., and Bjork, R. A. (2005). Illusions of competence in monitoring one's knowledge during study. J. Exp. Psychol. Learn. Memory Cogn. 31, 187-194. doi: 10.1037/0278-7393.31.2.187 also like to thank James Harnsberger and the Springfield College Office of Academic Affairs for financial support and the Springfield College Athletic Counseling Research Team whose feedback facilitated completion of the manuscript in a timely manner.

Kühn, S., Fernyhough, C., Alderson-Day, B., and Hurlburt, R. T. (2014). Inner experience in the scanner: can high fidelity apprehensions of inner experience be integrated with fMRI? Front. Psychol. Cogn. Sci. 5:1393. doi: 10.3389/fpsyg.2014.01393

Landers, D. M. (1995). Sport psychology: the formative years, 1950-1980. Sport Psychol. 9, 406-417. doi: 10.1123/tsp.9.4.406

Lapping-Carr, L. R., and Heavey, C. L. (2017). Pristine inner experience and descriptive experience sampling: implications for psychology. Front. Psychol. 8:2170. doi: 10.3389/fpsyg.2017.02170

Larrain, A., and Haye, A. (2012). The discursive nature of inner speech. Theory Psychol. 22, 3-22. doi: 10.1177/0959354311423864

McKelvie, S. J. (2019). Classical introspection revisited: implications of research on visual imagery for the functions of pristine inner experience as apprehended by descriptive experience sampling. Curr. Psychol. doi: 10.1007/s12144-019-00176-2. [Epub ahead of print].

McPherson, S. L. (1999). Expert-novice differences in performance skills and problem representations of youth and adults during tennis competition. Res. Q. Exerc. Sport 70, 233-251. doi: 10.1080/02701367.1999. 10608043

Puchalska-Wasyl, M. M. (2016). Coalition and opposition in myself? On integrative and confrontational internal dialogs, their functions, and the types of inner interlocutors. J. Constr. Psychol. 29, 197-218. doi: 10.1080/10720537.2015.1084601

Puchalska-Wasyl, M. M. (2017). Relationship of personality with integration and confrontation in internal dialogues. Scand. J. Psychol. 58, 451-457. doi: 10.1111/sjop.12387

Thibodeaux, J., and Winsler, A. (2018). What do youth tennis athletes say to themselves? Observed and self-reported self-talk on the court. Psychol. Sport Exerc. 38, 126-136. doi: 10.1016/j.psychsport.2018.06.006

Van Raalte, J. L., Cornelius, A. E., Copeskey, M. K., and Brewer, B. W. (2014). Say what? An analysis of spontaneous self-talk categorization. Sport Psychol. 28, 390-393. doi: 10.1123/tsp.2014-0017

Van Raalte, J. L., and Vincent, A. (2017). "Self-Talk in Sport and Performance," in Oxford Research Encyclopedia of Psychology, ed O. Braddick (New York, NY: American Psychological Association \& Oxford University Press).

Van Raalte, J. L., Vincent, A., and Brewer, B. W. (2016). Self-talk: review and sport-specific model. Psychol. Sport Exerc. 22, 139-148. doi: 10.1016/j.psychsport.2015.08.004

Wells, G. L., and Loftus, E. F. (2003). "Eyewitness memory for people and events," in Handbook of Psychology: Forensic Psychology ed A. M. Goldstein (New York, NY: Wiley \& Sons ), 149-160

Whitehead, A. E., Taylor, J. A., and Polman, R. C. J. (2015). Evidence for skill level differences in the thought processes of golfers during high and low pressure situations. Front. Psychol. 6:1974. doi: 10.3389/fpsyg.2015. 01974

Conflict of Interest Statement: The authors declare that the research was conducted in the absence of any commercial or financial relationships that could be construed as a potential conflict of interest.

Copyright (c) 2019 Van Raalte, Vincent and Dickens. This is an open-access article distributed under the terms of the Creative Commons Attribution License (CC BY). The use, distribution or reproduction in other forums is permitted, provided the original author(s) and the copyright owner(s) are credited and that the original publication in this journal is cited, in accordance with accepted academic practice. No use, distribution or reproduction is permitted which does not comply with these terms. 